\title{
Depth and regularity modulo a principal ideal
}

\author{
Giulio Caviglia ${ }^{1}$ - Huy Tài Hà ${ }^{2}$ - Jürgen Herzog ${ }^{3}$ • \\ Manoj Kummini $^{4}$ - Naoki Terai ${ }^{5}$ - Ngo Viet Trung ${ }^{6}$
}

\section{Dedicated to Craig Huneke on the occasion of his 65th birthday}

Received: 29 June 2017 / Accepted: 13 January 2018 / Published online: 30 January 2018

C Springer Science+Business Media, LLC, part of Springer Nature 2018

\begin{abstract}
We study the relationship between depth and regularity of a homogeneous ideal $I$ and those of $(I, f)$ and $I: f$, where $f$ is a linear form or a monomial. Our results have several interesting consequences on depth and regularity of edge ideals of hypergraphs and of powers of ideals.
\end{abstract}

\section{Ngo Viet Trung}

nvtrung@math.ac.vn

Giulio Caviglia

gcavigli@math.purdue.edu

Huy Tài Hà

tha@tulane.edu

Jürgen Herzog

juergen.herzog@gmail.com

Manoj Kummini

mkummini@cmi.ac.in

Naoki Terai

terai@cc.saga-u.ac.jp

1 Department of Mathematics, Purdue University, 150 N. University Street, West Lafayette, IN 47907-2067, USA

2 Department of Mathematics, Tulane University, 6823 St. Charles Ave., New Orleans, LA 70118, USA

3 Fachbereich 6, Mathematik, Universität Duisburg-Essen, Campus Essen, 45117 Essen, Germany

4 Chennai Mathematical Institute, Siruseri, Tamilnadu 603103, India

5 Faculty of Culture and Education, Saga University, Saga 8408502, Japan

6 Institute of Mathematics, Vietnam Academy of Science and Technology, 18 Hoang Quoc Viet, Hanoi, Vietnam 
Keywords Depth · Regularity · Monomial ideal · Powers of an ideal · Edge ideal · Very well-covered graph $\cdot$ Chordal graph

Mathematics Subject Classification Primary 13C20; Secondary 13D45 - 14B05 . $05 \mathrm{C} 65$

\section{Introduction}

Let $R=k\left[x_{1}, \ldots, x_{n}\right]$ be a polynomial ring over a field $k$ and let $X=\operatorname{Proj} R / I$ be a projective scheme in $\mathbb{P}^{n-1}$, where $I$ is a homogeneous ideal. The main goal of our work is to understand the connection between algebraic invariants and properties of $X$ and its hypersurface sections. This is a classical topic that has inspired many important results in both algebraic geometry and commutative algebra. We shall focus on invariants that govern the computational complexity, namely the depth and the Castelnuovo-Mumford regularity (or simply, regularity). More specifically, we shall examine the relationship between the depth and the regularity of $I$ and $(I, f)$, and their powers, when $f \in R$ is a linear form or when $I$ is a monomial ideal and $f$ is a monomial.

Our work is inspired by a recent paper of Dao, Huneke and Schweig [9], in which it was proved that if $I \subseteq R$ is a monomial ideal and $x$ is a variable appearing in the generators of $I$ then

$$
\operatorname{reg} R / I \in\{\operatorname{reg} R /(I: x)+1, \operatorname{reg} R /(I, x)\}
$$

and if, in addition, $I$ is a squarefree monomial ideal then

$$
\text { depth } R / I \in\{\operatorname{depth} R /(I: x) \text {, depth } R /(I, x)\} \text {. }
$$

These results give recursive formulas for regularity and depth of squarefree monomial ideals and played a key role in their work on edge ideals of graphs.

Our first result extends these formulas of Dao, Huneke and Schweig to a large class of homogeneous ideals.

Theorem 3.1 Let $I=J+f H$, where $J$ and $H$ are homogeneous ideals in $R$ and $f$ is a linear form that is a non-zerodivisor of $R / J$. Then

(i) depth $R / I \in\{\operatorname{depth} R /(I: f)$, depth $R /(I, f)\}$.

(ii) $\operatorname{reg} R / I \in\{\operatorname{reg} R /(I: f)+1, \operatorname{reg} R /(I, f)\}$.

Note that $I: f=J+H$ and $(I, f)=(J, f)$ under the assumptions of Theorem 3.1. We also obtain similar formulas for the case where $f$ is any form of higher degree that is a non-zerodivisor of $R / J$.

Obviously, we can choose $I$ to be any monomial ideal and $f$ to be any variable. This particularly shows that the depth formula of Dao, Huneke and Schweig also holds for arbitrary monomial ideals. We can even extend their depth formula to the case $f$ being an arbitrary monomial as follows. 
Theorem 4.3 Let $I$ be a monomial ideal and let $f$ be an arbitrary monomial in $R$. Then

(i) depth $R / I \in\{\operatorname{depth} R /(I: f)$, depth $R /(I, f)\}$,

(ii) depth $R / I=\operatorname{depth} R /(I: f)$ if depth $R /(I, f) \geq \operatorname{depth} R /(I: f)$.

We will give examples showing that Theorem 4.3 is no longer true if $I$ is not a monomial ideal or if $f$ is not a monomial, and that there is no similar formula for $\operatorname{reg} R / I$ if $f$ is not a variable.

We can also make the regularity formula of Dao, Huneke and Schweig more precise as follows.

Theorem 4.7 Let $I$ be a monomial ideal and $x$ a variable of $R$. Then

(i) $\operatorname{reg} R / I=\operatorname{reg} R /(I: x)+1$ if $\operatorname{reg} R /(I: x)>R /(I, x)$,

(ii) $\operatorname{reg} R / I \in\{\operatorname{reg} R /(I, x)+1, \operatorname{reg} R /(I, x)\}$ if $\operatorname{reg} R /(I: x)=\operatorname{reg} R /(I, x)$,

(iii) $\operatorname{reg} R / I=\operatorname{reg} R /(I, x)$ if $\operatorname{reg} R /(I: x)<\operatorname{reg} R /(I, x)$.

From Theorem 4.7, it follows that $\operatorname{reg} I$ is either $\max \{\operatorname{reg}(I: x), \operatorname{reg}(I, x)\}$ or $\max \{\operatorname{reg}(I: x), \operatorname{reg}(I, x)\}+1$. In particular, if $\operatorname{reg}(I: x) \leq r$ for all variables $x$ of $R$, then reg $I \leq r+1$. This implication is very useful because one can use induction to estimate the regularity of a monomial ideal.

Our approach can be used to study the behavior of the functions depth $R / I^{t}$ and $\operatorname{reg} R / I^{t}$ for $t \geq 1$. If $I$ is the edge ideal of a hypergraph which contains a good leaf and $f$ is the monomial of the associated leaf, we show that $I^{t+1}: f=I^{t}$ and $\overline{I^{t+1}}: f=\overline{I^{t}}$ for all $t \geq 1$, where $\overline{I^{t}}$ denotes the integral closure of $I^{t}$. Using these relations, we derive the following result.

Theorem 5.1 Let $I$ be the edge ideal of a hypergraph which contains a good leaf. Then

(i) the functions depth $R / I^{t}$ and depth $R / \overline{I^{t}}$ are non-increasing for all $t \geq 1$,

(ii) the functions reg $R / I^{t}$ and $\operatorname{reg} R / \overline{I^{t}}$ are non-decreasing for all $t \geq 1$.

Notice that the function depth $R / I^{t}$ of the edge ideal of a hypergraph needs not be non-increasing (see [16,23]), and that it is still an open question (see [1]) whether the depth function of the edge ideal of a graph is non-increasing.

In general, the functions depth $R / I^{t}$ and reg $R / I^{t}$ need not be monotone; see [1,2, $14,15]$. However, if we can find a common non-zerodivisor $f$ on $I^{t}$ for all $t \geq 1$, then depth $R /(I, f)^{t}$ and reg $R /(I, f)^{t}$ are monotone functions regardless of the behavior of depth $R / I^{t}$ and reg $R / I^{t}$. This follows from the following result, whose proof is based on the same ideas as that of Theorem 3.1.

Theorem 5.2 Let $R$ be a positively graded algebra over a field, and let $I$ be a graded ideal in $R$. Let $f \neq 0$ be a form in $R$ which is a non-zerodivisor of $R / I^{t}$ for all $t \leq s$. Then

(i) depth $R /(I, f)^{s}=\min _{t \leq s} \operatorname{depth} R / I^{t}-1$,

(ii) $\operatorname{reg} R /(I, f)^{s}-s \operatorname{deg} f=\max _{t \leq s}\left\{\operatorname{reg} R / I^{t}-t \operatorname{deg} f\right\}$.

As a consequence, if $f=x$ is a new variable then depth $R[x] /(I, x)^{s}$ is a non-increasing function and $\operatorname{reg} R[x] /(I, x)^{s}$ is a non-decreasing function, while the functions depth $R / I^{S}$ and $\operatorname{reg} R / I^{S}$ need not be so. 
The relationship between depth and regularity of the ideals $I, I: x,(I, x)$ is very useful in finding combinatorial characterizations of these invariants for edge ideals of hypergraphs. We will demonstrate this approach in two applications.

Let $I(\mathcal{H})$ denote the edge ideal of a hypergraph $\mathcal{H}$ in a polynomial ring $R$. We call $\mathcal{H}$ a Cohen-Macaulay hypergraph if $R / I(\mathcal{H})$ is a Cohen-Macaulay ring, i.e., $\operatorname{depth} R / I(\mathcal{H})=\operatorname{dim} R / I(\mathcal{H})$. Moreover, for simplicity, we set reg $\mathcal{H}=\operatorname{reg} I(\mathcal{H})$ and call it the regularity of $\mathcal{H}$.

The first application is a short proof for the following characterization of CohenMacaulay very well-covered graphs, which is essentially due to Crupi, Rinaldo and Terai [7] (see also Constantinescu and Varbaro [6] and Mahmoudi et al [27] for other variants of this characterization). This characterization is a generalization of a criterion for Cohen-Macaulay bipartite graphs of Herzog and Hibi [18].

Theorem 6.3 Let $\mathcal{H}$ be a simple graph on $2 n$ vertices which has a minimal vertex cover of $n$ elements. Then $\mathcal{H}$ is a Cohen-Macaulay graph if and only if $\mathcal{H}$ is a twin-free very well-covered graph.

The second application is a sufficient condition for a hypergraph to have regularity $\leq 3$. Since the maximal generating degree of a homogeneous ideal is bounded above by its regularity, the edge ideal of a hypergraph of regularity 2 is that of a graph. Fröberg [13] characterized graphs of regularity $\leq 2$ to be those whose complements are chordal.

For every vertex $x$ of a hypergraph $\mathcal{H}$, we denote by $\mathcal{H}: x$ the hypergraph of all minimal sets of the form $F \cap(V \backslash\{x\})$, where $F$ is an edge of $\mathcal{H}$.

Theorem 6.4 Let $\mathcal{H}$ be a hypergraph such that $\mathcal{H}: x$ is a graph whose complement is chordal for all vertices $x$ of $\mathcal{H}$. Then $\operatorname{reg} \mathcal{H} \leq 3$.

A special case of Theorem 6.4 is a result of Nevo on the regularity of gap-free and claw-free graphs [30], which was also reproved by Dao, Huneke and Schweig in [9].

In publishing this paper, we hope that our results and methods would stimulate further investigations on the behavior of depth and regularity between the ideals $I$, $I: f$ and $(I, f)$.

\section{Preliminaries}

In this section, we recall notations and terminology used in the paper, and a number of auxiliary results. Generally, we will follow standard texts in this research area (cf. $[5,10,19])$.

Throughout the paper, the two important invariants that we investigate are the depth and the regularity. Though these notions can be defined in several ways, we prefer their definition by means of local cohomology modules.

Let $R$ be a positively graded algebra and let $\mathfrak{m}$ be its maximal homogeneous ideal. Let $M$ be a finitely generated graded $R$-module. Let $H_{\mathfrak{m}}^{i}(M)$, for $i \geq 0$, denote the $i$-th local cohomology module of $M$ with respect to $\mathfrak{m}$. We define 


$$
\begin{aligned}
\operatorname{depth} M & :=\min \left\{i \mid H_{\mathfrak{m}}^{i}(M) \neq 0\right\}, \\
\operatorname{reg} M & :=\max \left\{i+j \mid H_{\mathfrak{m}}^{i}(M)_{j} \neq 0\right\} .
\end{aligned}
$$

Remark 2.1 Let $a_{i}(M):=\max \left\{j \mid H_{\mathfrak{m}}^{i}(M)_{j} \neq 0\right\}$ with the convention that $a_{i}(M):=$ $-\infty$ if $H_{\mathfrak{m}}^{i}(M)=0$. Then

$$
\begin{aligned}
\operatorname{depth} M & =\min \left\{i \mid a_{i}(M) \neq-\infty\right\}, \\
\operatorname{reg} M & =\max \left\{a_{i}(M)+i \mid i \geq 0\right\} .
\end{aligned}
$$

where $R$ is a polynomial ring, $M$ is a finitely generated graded $R$-module, and the depth and the regularity of $M$ are closely related to the minimal free resolution and graded Betti numbers of $M$ in the following way. Suppose that $M$ admits the following minimal free resolution:

$$
0 \rightarrow \bigoplus_{j \in \mathbb{Z}} R(-j)^{\beta_{p, j}(M)} \rightarrow \cdots \rightarrow \bigoplus_{j \in \mathbb{Z}} R(-j)^{\beta_{0, j}(M)} \rightarrow M \rightarrow 0
$$

Let $\mathrm{pd} M$ denote the projective dimension of $M$. Then

$$
\begin{aligned}
\operatorname{pd} M & =\max \left\{i \mid \beta_{i, j}(M) \neq 0 \text { for some } j \in \mathbb{Z}\right\}, \\
\operatorname{depth} M & =n-\operatorname{pd} M, \\
\operatorname{reg} M & =\max \left\{j-i \mid \beta_{i, j}(M) \neq 0\right\} .
\end{aligned}
$$

Note that the maximal degree of the minimal generators of $M$ is bounded by reg $M$.

The definition of depth and regularity by means of the local cohomology modules is best suited when working with short exact sequences. In fact, using the long derived sequence of local cohomology modules, we immediately obtain the following facts, which we shall need later in the next sections.

Lemma 2.2 Let $0 \rightarrow L \rightarrow M \rightarrow N \rightarrow 0$ be a short exact sequence of finite graded $R$-modules. Then

(i) depth $M \geq \min \{\operatorname{depth} L$, depth $N\}$,

(ii) depth $M=\operatorname{depth} N$ if $\operatorname{depth} L>\operatorname{depth} M$,

(iii) $\operatorname{reg} L=\operatorname{reg} N+1$ if $\operatorname{reg} L>\operatorname{reg} M$.

From now on, unless otherwise stated, we let $R=k\left[x_{1}, \ldots, x_{n}\right]$ be a polynomial ring over a field $k$ and let $I$ be a nonzero homogeneous ideal in $R$. From the above characterizations of depth and regularity by means of the graded Betti numbers we immediately have the following relationships between $R / I$ and $I$ :

$$
\begin{aligned}
\operatorname{depth} R / I & =\operatorname{depth} I-1, \\
& \operatorname{reg} R / I=\operatorname{reg} I-1 .
\end{aligned}
$$

These formulas will be used without reference in this paper.

Recall that for a finitely generated graded $R$-module $M$, an element $f \in R$ is called a filter-regular element of $M$ if $f \notin \mathfrak{p}$ for all associated primes $\mathfrak{p} \neq \mathfrak{m}$ of $M$. A 
sequence $f_{1}, \ldots, f_{s} \in R$ of elements is called a filter-regular sequence of $M$ if $f_{i}$ is a filter-regular element of $M /\left(f_{1}, \ldots, f_{i-1}\right) M$ for all $i=1, \ldots, s$. It is easy to see that this is equivalent to the condition that

$$
\left[\left(f_{1}, \ldots, f_{i-1}\right) M: f_{i}\right]_{t}=\left[\left(f_{1}, \ldots, f_{i-1}\right) M\right]_{t}
$$

for all $i=1, \ldots, s$, and $t \gg 0$ (see [34, Lemma 2.1]).

Remark 2.3 If $k$ is an infinite field, we can use prime avoidance to find a linear form which is filter-regular with respect to $M$. Applying this fact successively, we may assume that $x_{n}, \ldots, x_{1}$ is a filter-regular sequence of $M$ after a linear change of the variables.

Let in $(I)$ denote the initial ideal of $I$ with respect to the degree reverse lexicographic order. When the $x_{n}, \ldots, x_{1}$ is a filter-regular sequence of $R / I$, one can easily pass the investigation from $I$ to in $(I)$.

Theorem 2.4 Assume that $x_{n}, \ldots, x_{1}$ is a filter-regular sequence of $R / I$. Then

(i) depth $R / I=\operatorname{depth} R / \operatorname{in}(I)$,

(ii) $\operatorname{reg} R / I=\operatorname{reg} R / \operatorname{in}(I)$.

Proof This follows from Remark 2.1 and a general result [35, Theorem 1.2], which states that

$$
\max \left\{a_{i}(R / I)+i \mid i \leq t\right\}=\max \left\{a_{i}(R / \operatorname{in}(I))+i \mid i \leq t\right\}
$$

for every $t \geq 0$.

Our investigation on monomial ideals is based on a formula of Takayama. Let $R=$ $k\left[x_{1}, \ldots, x_{n}\right]$ and $I$ a monomial ideal in $R$. Then $R / I$ has a natural $\mathbb{N}^{n}$-graded structure inherited from that of $R$. Therefore, the local cohomology module $H_{\mathfrak{m}}^{i}(R / I)$ has a $\mathbb{Z}^{n}$-graded structure. Takayama's formula [33, Theorem 1] describes the dimension of the $\mathbb{Z}^{n}$-graded component $H_{\mathfrak{m}}^{i}(R / I) \mathbf{a}$, for $\mathbf{a} \in \mathbb{Z}^{n}$, in terms of a simplicial complexes $\Delta_{\mathbf{a}}(I)$. We shall recall the construction of $\Delta_{\mathbf{a}}(I)$, as given in [29], which is simpler than the original construction of [33].

For $\mathbf{a}=\left(a_{1}, \ldots, a_{n}\right) \in \mathbb{Z}^{n}$, set $x^{\mathbf{a}}=x_{1}^{a_{1}} \cdots x_{n}^{a_{n}}$ and $G_{\mathbf{a}}:=\left\{j \in[1, n] \mid a_{j}<0\right\}$. For every subset $F \subseteq[1, n]$, let $R_{F}=R\left[x_{j}^{-1} \mid j \in F\right]$. Define

$$
\Delta_{\mathbf{a}}(I)=\left\{F \backslash G_{\mathbf{a}} \mid G_{\mathbf{a}} \subseteq F, x^{\mathbf{a}} \notin I R_{F}\right\}
$$

We call $\Delta_{\mathbf{a}}(I)$ a degree complex of $I$.

Takayama's formula is stated as follows:

$$
\operatorname{dim}_{k} H_{\mathfrak{m}}^{i}(R / I)_{\mathbf{a}}=\operatorname{dim}_{k} \widetilde{H}_{i-\left|G_{\mathbf{a}}\right|-1}\left(\Delta_{\mathbf{a}}(I), k\right) .
$$

The original formula in [33, Theorem 1] is slightly different. It contains additional conditions on a for $H_{\mathfrak{m}}^{i}(R / I)_{\mathbf{a}}=0$. However, the proof in [33] shows that we may drop these conditions, which is more convenient for our investigation. 
From Takayama's formula, we immediately obtain the following characterizations of depth and regularity of monomial ideals in terms of the degree complexes.

Proposition 2.5 Let $I \subseteq R$ be a monomial ideal. Then

(i) depth $R / I=\min \left\{\left|G_{\mathbf{a}}\right|+i \mid \mathbf{a} \in \mathbb{Z}^{n}, i \geq 0, \widetilde{H}_{i-1}\left(\Delta_{\mathbf{a}}(I), k\right) \neq 0\right\}$,

(ii) $\operatorname{reg} R / I=\max \left\{|\mathbf{a}|+\left|G_{\mathbf{a}}\right|+i \mid \mathbf{a} \in \mathbb{Z}^{n}, i \geq 0, \widetilde{H}_{i-1}\left(\Delta_{\mathbf{a}}(I), k\right) \neq 0\right\}$.

\section{Depth and regularity modulo a linear form}

The main aim of this section is to prove the following theorem, which extends the aforementioned results of Dao, Huneke and Schweig on monomial ideals to a large class of homogeneous ideals. Unless otherwise specified, let $R=k\left[x_{1}, \ldots, x_{n}\right]$ be a polynomial ring over a field and let $I$ be a homogeneous ideal in $R$.

Theorem 3.1 Let $I=J+f H$, where $J$ and $H$ are homogeneous ideals in $R$ and $f$ is a linear form that is a non-zerodivisor of $R / J$. Then

(i) depth $R / I \in\{\operatorname{depth} R /(J+H)$, depth $R / J-1\}$.

(ii) $\operatorname{reg} R / I \in\{\operatorname{reg} R /(J+H)+1, \operatorname{reg} R / J\}$.

Proof Let $S=R[x]$, where $x$ is a new variable, and $Q=(J, x H)$. Then

$$
R / I=S /(Q, x-f) .
$$

We shall show that $x-f$ is a non-zerodivisor in $S / Q$. Indeed, it is clear that $Q=(J, x) \cap(J, H)$. Observe that every associated prime of $(J, x)$ is of the form $(\mathfrak{p}, x)$, where $\mathfrak{p}$ is an associated prime of $J$, and $f \notin \mathfrak{p}$ because $f$ is a non-zerodivisor in $R / J$. Thus, $x-f$ does not belong to any associated prime of $(J, x)$. Hence, $(J, x):(x-f)=(J, x)$. Since every associated prime of $(J, H)$ is the extension to $S$ of an associated prime of $J+H \subseteq R, x-f$ does not belong to any associated prime of $(J, H)$. Therefore, $(J, H):(x-f)=(J, H)$. Hence, we get $Q:(x-f)=Q$.

It follows from the fact that $x-f$ is a non-zerodivisor of $S / Q$ that

$$
\text { depth } R / I=\operatorname{depth} S / Q-1=\operatorname{depth} Q-2=\operatorname{depth} Q / x Q-1 \text {. }
$$

It is easy to see that $Q / x Q=J \oplus((J+H) / J)(-1)$. Thus,

$$
\operatorname{depth} Q / x Q=\min \{\operatorname{depth} J, \operatorname{depth}(J+H) / J\} \text {. }
$$

By Lemma 2.2(i), from the exact sequence

$$
0 \rightarrow J \rightarrow(J+H) \rightarrow(J+H) / J \rightarrow 0
$$

we obtain

$$
\operatorname{depth}(J+H) \geq \min \{\operatorname{depth} J, \operatorname{depth}(J+H) / J\} .
$$


Therefore,

depth $R / I=\operatorname{depth} Q / x Q-1 \leq \operatorname{depth}(J+H)-1=\operatorname{depth} R /(J+H)$.

Now, if depth $R / I \neq \operatorname{depth} R /(J+H)$ then it follows that

$$
\operatorname{depth} R / I<\operatorname{depth} R /(J+H)=\operatorname{depth} R / I: f \text {. }
$$

Applying Lemma 2.2(ii) to the exact sequence

$$
0 \rightarrow R / I: f \stackrel{f}{\rightarrow} R / I \rightarrow R /(I, f) \rightarrow 0
$$

we get depth $R / I=\operatorname{depth} R /(I, f)=\operatorname{depth} R /(J, f)=\operatorname{depth} R / J-1$ as required for Theorem 3.1(i).

The proof for Theorem 3.1(ii) proceeds similarly. Firstly, we have

$$
\operatorname{reg} R / I=\operatorname{reg} S / Q=\operatorname{reg} Q-1=\operatorname{reg} Q / x Q-1 .
$$

On the other hand,

$$
\operatorname{reg} Q / x Q=\max \{\operatorname{reg} J, \operatorname{reg}(J+H) / J+1\}
$$

Therefore,

$$
\begin{aligned}
\operatorname{reg} R / I & =\max \{\operatorname{reg} J, \operatorname{reg}(J+H) / J+1\}-1 \\
& =\max \{\operatorname{reg} R / J, \operatorname{reg}(J+H) / J\} .
\end{aligned}
$$

If $\operatorname{reg} R / I \neq \operatorname{reg} R / J$ then

$$
\operatorname{reg} R / I=\operatorname{reg}(J+H) / J>\operatorname{reg} R / J .
$$

By Lemma 2.2(iii), from the short exact sequence

$$
0 \rightarrow(J+H) / J \rightarrow R / J \rightarrow R /(J+H) \rightarrow 0
$$

we get $\operatorname{reg}(J+H) / J=\operatorname{reg} R /(J+H)+1$. Hence, $\operatorname{reg} R / I=\operatorname{reg} R /(J+H)+1$ as required.

It is easy to see that $(I: f)=J+H$ and $(I, f)=(J, f)$ under the assumption of Theorem 3.1. Hence, we can rewrite the formulae of Theorem 3.1 as

(i) depth $R / I \in\{\operatorname{depth} R /(I: f)$, depth $R /(I, f)\}$,

(ii) $\operatorname{reg} R / I \in\{\operatorname{reg} R /(I: f)+1, \operatorname{reg} R /(I, f)\}$.

Our proof of Theorem 3.1 in fact yields a slightly more general statement where $f$ is of higher degree.

Corollary 3.2 Let $I=J+f H$, where $J$ and $H$ are homogeneous ideals in $R$ and $f$ is any form of degree $d$ that is a non-zerodivisor of $R / J$. Then 
(i) depth $R / I \in\{\operatorname{depth} R /(J+H)$, depth $R / J-1\}$.

(ii) $\operatorname{reg} R / I \in\{\operatorname{reg} R /(J+H)+d, \operatorname{reg} R / J+d-1, \ldots, \operatorname{reg} R / J\}$.

Proof As in the proof of Theorem 3.1, consider $S=R[x]$, where $x$ is a new variable, and $Q=\left(J, x^{d} H\right)$. The same argument shows that $x^{d}-f$ is a non-zerodivisor in $S / Q$. Thus, we may again reduce to the statements for $Q \subseteq S$. Observe that $Q=\left(J, x\left(x^{d-1} H\right)\right)$. The assertion now follows by an iterated application of Theorem 3.1 .

The assumption of Theorem 3.1 is obviously satisfied if $I$ is any monomial ideal and $f$ is any variable. Therefore, we have the following consequence.

Corollary 3.3 Let I be a monomial ideal and let $x$ be a variable in $R$. Then

(i) depth $R / I \in\{\operatorname{depth} R /(I: x)$, depth $R /(I, x)\}$,

(ii) $\operatorname{reg} R / I \in\{\operatorname{reg} R /(I: x)+1, \operatorname{reg} R /(I, x)\}$.

Note that Corollary 3.3(ii) was already proved by Dao, Huneke and Schweig [9, Lemma 2.10]. They also proved Corollary 3.3(i) for squarefree monomial ideals [9, Lemma 5.1]. Their proofs are based on a result of Kummini [25] on multigraded Betti numbers of squarefree monomial ideals.

The following examples show that Corollary 3.3 does not hold for an arbitrary linear form $f$.

Example 3.4 Let $I=\left(x_{1} x_{3}, x_{2} x_{3}, x_{1} x_{4}\right)$ and $f=x_{1}-x_{3}$ in $R=\mathbb{Q}\left[x_{1}, x_{2}, x_{3}, x_{4}\right]$. Then depth $R / I=2$, depth $R /(I: f)=1$ and depth $R /(I, f)=0$.

Example 3.5 Let $I=\left(x_{2}^{3}, x_{2} x_{3} x_{5}, x_{3} x_{8}^{2} x_{9}, x_{5} x_{7} x_{9}^{3}\right)$ and $f=x_{3}-x_{5}$ in $R=$ $\mathbb{Q}\left[x_{1}, \ldots, x_{9}\right]$. Then $\operatorname{reg} R / I=6, \operatorname{reg} R /(I: f)=7$ and $\operatorname{reg} R /(I, f)=7$.

Under the assumption that $f$ is general enough, Theorem 3.1 gives the following result.

Corollary 3.6 Let $f \in R$ be a linear form, which is filter-regular in $R / I$. Then

(i) depth $R / I \in\{\operatorname{depth} R /(I: f)$, depth $R /(I, f)\}$.

(ii) $\operatorname{reg} R / I \in\{\operatorname{reg} R /(I: f)+1, \operatorname{reg} R /(I, f)\}$.

Proof Without restriction we may assume that $k$ is an infinite field. Using prime avoidance and a linear change of variables, we may assume that $f=x_{n}$ and

(1) $x_{n}, \ldots, x_{1}$ form a filter-regular sequence in $R / I$,

(2) $x_{n}, \ldots, x_{1}$ form a filter-sequence in $R /\left(I: x_{n}\right)$,

(3) $x_{n-1}, \ldots, x_{1}$ form a filter-sequence in $R /\left(I, x_{n}\right)$.

Let in $(I)$ denote the initial ideal of $I$ with respect to the reverse lexicographic order. By Theorem 2.4, we have

(4) depth $R / I=\operatorname{depth} R / \operatorname{in}(I)$ and $\operatorname{reg} R / I=\operatorname{reg} R / \operatorname{in}(I)$,

(5) depth $R / I: x_{n}=\operatorname{depth} R / \operatorname{in}\left(I: x_{1}\right)$ and $\operatorname{reg} R / I: x_{n}=\operatorname{reg} R / \operatorname{in}\left(I: x_{n}\right)$,

(6) depth $R /\left(I, x_{n}\right)=\operatorname{depth} R / \operatorname{in}\left(I, x_{n}\right)$ and $\operatorname{reg} R /\left(I, x_{n}\right)=\operatorname{reg} R / \operatorname{in}\left(I, x_{n}\right)$. 
It is also known (see [10, Proposition 15.12], [19, Lemma 4.3.7]) that

(7) in $\left(I: x_{n}\right)=\operatorname{in}(I): x_{n}$,

(8) in $\left(I, x_{n}\right)=$ in $(I)+\left(x_{n}\right)$.

Since in $(I)$ is a monomial ideal, there are monomial ideals $J$ and $H$ such that in $(I)=$ $J+x_{n} H$ and $x_{n}$ is a non-zerodivisor in $R / J$. Obviously, we have

(9) in $(I): x_{n}=J+H$,

(10) in $(I)+\left(x_{n}\right)=\left(J, x_{n}\right)$.

Using (4)-(10), we can express the asserted formulas as

$$
\begin{gathered}
\operatorname{depth} R / \operatorname{in}(I) \in\{\operatorname{depth} R /(J+H), \operatorname{depth} R / J-1\}, \\
\operatorname{reg} R / \operatorname{in}(I) \in\{\operatorname{reg} R /(J+H)+1, \operatorname{reg} R / J\} .
\end{gathered}
$$

The conclusion now follows from Theorem 3.1.

Corollary 3.6 is only interesting in the case depth $R / I=0$. If depth $R / I>0$, then $f$ must be a regular element; hence, depth $R / I=\operatorname{depth} R /(I: f)$ and $\operatorname{reg} R / I=$ $\operatorname{reg} R /(I, f)$.

\section{Depth and regularity colon a monomial}

It is natural to ask whether one can extend the results of Dao, Huneke and Schweig to the case where $x$ is replaced by a form $f$ of higher degree. We shall see that the same relationship between the depths of $R / I, R /(I: f)$ and $R /(I, f)$ as in Corollary 3.3(i) holds for any monomial ideal $I$ and any monomial $f$. We shall also give examples showing that this is no longer true if $I$ is not a monomial ideal or if $f$ is not a monomial, and that there is no similar relationship between the regularities of $R / I, R /(I: f)$ and $R /(I, f)$ as in Corollary 3.3(ii).

The investigation in this section is based on the following relationships between $R / I$ and $R /(I: f)$, which were proved in [31, Corollary 1.3] for (i) and in [32, Lemma 4.2] for (ii) by different methods.

Lemma 4.1 Let I be a monomial ideal and let $f$ be an arbitrary monomial in $R$. Then

(i) depth $R / I \leq \operatorname{depth} R /(I: f)$,

(ii) $\operatorname{reg} R / I \geq \operatorname{reg} R /(I: f)$.

Proof (i) By Proposition 2.5(i), there exists a $\in \mathbb{Z}^{n}$ such that depth $R /(I: f)=$ $\left|G_{\mathbf{a}}\right|+i$ and $\tilde{H}_{i-1}\left(\Delta_{\mathbf{a}}(I: f), k\right) \neq 0$ for some $i \geq 0$. Let $f=x^{\mathbf{b}}, \mathbf{b} \in \mathbb{N}^{n}$. Since $G_{\mathbf{a}}:=\left\{j \mid a_{j}<0\right\}$, there exists $\mathbf{c} \in \mathbb{N}^{n} \operatorname{such}$ that $\operatorname{supp}(\mathbf{c}) \subseteq G_{\mathbf{a}}$ and $G_{\mathbf{a}+\mathbf{b}-\mathbf{c}}=G_{\mathbf{a}}$. For every subset $F \subset[1, n], F \supseteq G_{\mathbf{a}}$, the element $x$ is invertible in $R_{F}:=k\left[x_{i}^{-1} \mid i \in F\right]$. Therefore, $x^{\mathbf{a}} \in(I: f) R_{F}$ if and only if $x^{\mathbf{a}+\mathbf{b}} \in I R_{F}$ if and only if $x^{\mathbf{a}+\mathbf{b}-\mathbf{c}} \in I R_{F}$. By the definition of the degree complex, this implies that $\Delta_{\mathbf{a}}(I: f)=\Delta_{\mathbf{a}+\mathbf{b}-\mathbf{c}}(I)$. Hence, $\tilde{H}_{i-1}\left(\Delta_{\mathbf{a}+\mathbf{b}-\mathbf{c}}(I), k\right) \neq 0$. By Proposition 2.5 (i), it follows that

$$
\operatorname{depth} R / I \leq\left|G_{\mathbf{a}+\mathbf{b}-\mathbf{c}}\right|+i=\left|G_{\mathbf{a}}\right|+i=\operatorname{depth} R /(I: f) \text {. }
$$


(ii) By Proposition 2.5(ii), there exists $\mathbf{a} \in \mathbb{Z}^{n}$ such that $\operatorname{reg}(I: f)=|\mathbf{a}|+\left|G_{\mathbf{a}}\right|+i$ and $\tilde{H}_{i-1}\left(\Delta_{\mathbf{a}}(I: f), k\right) \neq 0$ for some $i \geq 0$. By the maximality of $|\mathbf{a}|+\left|G_{\mathbf{a}}\right|+i$ we must have $a_{j}=-1$ for $j \in G_{\mathbf{a}}$. Let $\mathbf{c} \in \mathbb{N}^{n}$ with $c_{i}=b_{i}$ for $i \in G_{\mathbf{a}}$ and $c_{i}=0$ else. Then $\operatorname{supp}(\mathbf{c}) \subseteq G_{\mathbf{a}}$ and $G_{\mathbf{a}+\mathbf{b}-\mathbf{c}}=G_{\mathbf{a}}$. Moreover, $|\mathbf{a}+\mathbf{b}-\mathbf{c}| \geq|\mathbf{a}|$. Similarly as above, we have $\Delta_{\mathbf{a}}(I: f)=\Delta_{\mathbf{a}+\mathbf{b}-\mathbf{c}}(I)$. Hence, $\tilde{H}_{i-1}\left(\Delta_{\mathbf{a}+\mathbf{b}-\mathbf{c}}(I), k\right) \neq 0$. By Proposition 2.5(ii), this implies that

$$
\operatorname{reg} R / I \geq|\mathbf{a}+\mathbf{b}-\mathbf{c}|+\left|G_{\mathbf{a}}\right|+i \geq|\mathbf{a}|+\left|G_{\mathbf{a}}\right|+i=\operatorname{reg} R /(I: f) .
$$

One might expect that $\operatorname{reg} R / I \geq \operatorname{reg} R /(I: f)+\operatorname{deg} f$. As shown by the following example, this bound does not hold, even when $f$ is a variable appearing in the generators of $I$.

Example 4.2 Let $R=\mathbb{Q}\left[x_{1}, \ldots, x_{9}\right]$ and consider

$$
I=\left(x_{2} x_{3}, x_{2} x_{4}, x_{3} x_{5}, x_{4} x_{5}, x_{2} x_{5} x_{7}, x_{4} x_{8}, x_{3} x_{9}, x_{4} x_{9}, x_{7} x_{9}, x_{8} x_{9}\right) .
$$

Then, for $f=x_{8}$, we have $\operatorname{reg} R / I=2=\operatorname{reg} R /(I: f)$. Thus, $\operatorname{reg} R / I \nsupseteq \operatorname{reg} R /(I$ : $f)+1$.

Our next result shows that the conclusion of Corollary 3.3(i) also holds for an arbitrary monomial $f$.

Theorem 4.3 Let I be a monomial ideal and let $f$ be an arbitrary monomial in $R$. Then

(i) depth $R / I \in\{$ depth $R /(I: f)$, depth $R /(I, f)\}$.

(ii) depth $R / I=\operatorname{depth} R /(I: f)$ if depth $R /(I, f) \geq \operatorname{depth} R /(I: f)$.

Proof Assume that depth $R / I \neq \operatorname{depth} R /(I: f)$. Then $\operatorname{depth} R /(I: f)>$ depth $R / I$ by Lemma 4.1(i). Consider the short exact sequence

$$
0 \longrightarrow R /(I: f) \stackrel{f}{\longrightarrow} R / I \longrightarrow R /(I, f) \longrightarrow 0 .
$$

By Lemma 2.2(ii), this implies that depth $R / I=\operatorname{depth} R /(I, f)$.

If depth $R /(I, f) \geq \operatorname{depth} R /(I: f)$, then depth $R / I \geq \operatorname{depth} R /(I: f)$ by Lemma 2.2(i). By Lemma 4.1(i), this implies that depth $R / I=\operatorname{depth} R /(I: f)$.

Theorem 4.3(i) does not necessarily hold if $I$ is not a monomial ideal or if $f$ is not a monomial.

Example 4.4 Let $R=\mathbb{Q}[x, y, z, u, v]$ and consider

$$
I=\left(x^{2} y^{3}-z^{2} u^{3}, y^{2} z^{3}-x^{2} z u v, x^{3} u^{2}-y^{2} v^{3}\right) \text { and } f=x y z u v .
$$

Then depth $R / I=2$, depth $R /(I: f)=1$ and depth $R /(I, f)=0$. 
Example 4.5 Let $R=\mathbb{Q}[x, y, z, u, v]$ and consider

$$
I=\left(x^{3} y, y^{2} z^{5}, z^{2} u^{4} v\right) \text { and } f=x y-z v .
$$

Then depth $R / I=2$, depth $R /(I: f)=1$ and depth $R /(I, f)=0$.

From the short exact sequence $0 \longrightarrow R /(I: f) \stackrel{f}{\longrightarrow} R / I \longrightarrow R /(I, f) \longrightarrow 0$, we might expect that

$$
\operatorname{reg} R / I \in\{\operatorname{reg} R /(I: f)+\operatorname{deg} f, \operatorname{reg} R /(I, f)\}
$$

which would generalize the mentioned result of Dao, Huneke and Schweig [9, Lemma $2.10]$ to the case where $\operatorname{deg} f>1$. However, the following example shows that this is not possible, even when $I$ is a monomial ideal and $f$ is a monomial.

Example 4.6 Let $R=\mathbb{Q}[x, y, z, u, v]$ and consider

$$
I=\left(x y^{2}, y z^{2}, z u^{3}, u v^{2} x, v^{2} x z\right) \text { and } f=x^{3} y .
$$

Then $\operatorname{reg} R / I=6, \operatorname{reg} R /(I, f)=7$ and $\operatorname{reg} R /(I: f)+\operatorname{deg} f=8$. In particular, $\operatorname{reg} R / I \notin\{\operatorname{reg} R /(I: f)+\operatorname{deg} f, \operatorname{reg} R /(I, f)\}$.

On the other hand, the formula for reg $R / I$ of Dao, Huneke and Schweig can be made more precise as follows.

Theorem 4.7 Let I be a monomial ideal and $x$ a variable of $R$. Then

(i) $\operatorname{reg} R / I=\operatorname{reg} R /(I: x)+1$ if $\operatorname{reg} R /(I: x)>R /(I, x)$,

(ii) $\operatorname{reg} R / I \in\{\operatorname{reg} R /(I, x)+1, \operatorname{reg} R /(I, x)\}$ if $\operatorname{reg} R /(I: x)=\operatorname{reg} R /(I, x)$,

(iii) $\operatorname{reg} R / I=\operatorname{reg} R /(I, x)$ if $\operatorname{reg} R /(I: x)<\operatorname{reg} R /(I, x)$.

Proof We only need to prove (i) and (iii).

If $\operatorname{reg} R /(I: x)>\operatorname{reg} R /(I, x)$, then $\operatorname{reg} R / I>\operatorname{reg} R /(I, x)$ by Lemma 4.1(ii). Hence, $\operatorname{reg} R / I=\operatorname{reg} R /(I: x)+1$.

If $\operatorname{reg} R /(I: x)<\operatorname{reg} R /(I, x)$, then $\operatorname{reg} R / I>\operatorname{reg} R /(I: x)$ because reg $R / I \geq$ $\operatorname{reg} R /(I, x)$ by the proof of [9, Lemma 2.10]. Therefore, $\operatorname{reg} R / I=\operatorname{reg} R /(I, x)$.

From Theorem 4.7, we immediately obtain the following estimate, which shows that $\operatorname{reg} I$ is either $\max \{\operatorname{reg}(I: x), \operatorname{reg}(I, x)\}$ or $\max \{\operatorname{reg}(I: x), \operatorname{reg}(I, x)\}+1$.

Corollary 4.8 Let $I$ be a monomial ideal and $x$ a variable of $R$. Then

$$
\max \{\operatorname{reg}(I: x), \operatorname{reg}(I, x)\} \leq \operatorname{reg} I \leq \max \{\operatorname{reg}(I: x)+1, \operatorname{reg}(I, x)\}
$$

In particular, we obtain the following inductive estimate for the regularity of monomial ideals.

Proposition 4.9 Let $I$ be a monomial ideal. If $\operatorname{reg}(I: x) \leq r$ for all variables $x$ of $R$, then $\operatorname{reg} I \leq r+1$. 
Proof The case $n=1$ is trivial. If $n>1$, we have $(I, x): y=(I: y, x)$ for all variables $x, y$ of $R$. Therefore, $\operatorname{reg}((I, x): y)=\operatorname{reg}(I: y, x) \leq \operatorname{reg}(I: y) \leq r$ by the lower bound of Corollary 4.8. By induction, we have reg $(I, x) \leq r+1$. Therefore, the upper bound of Corollary 4.8 implies reg $I \leq r+1$.

Using Proposition 4.9, we can easily recover the following result.

Corollary 4.10 [22, Theorem 3.1] Let I be a monomial ideal. Let $1 \mathrm{~cm}(I)$ denote the least common multiple of the minimal monomial generators of I. Then

$$
\operatorname{reg} I \leq \operatorname{deg} \operatorname{lcm}(I)-\text { ht } I+1
$$

Proof The case $\operatorname{deg} \operatorname{lcm}(I)=1$ is trivial. Without restriction, we may assume that every variable $x$ appears in the minimal monomial generators of $I$. Then $\operatorname{deg} \operatorname{lcm}(I$ : $x) \leq \operatorname{deg} \operatorname{lcm}(I)-1$ and $h t(I: x) \geq$ ht $I$. By induction, we have

$$
\operatorname{reg}(I: x) \leq \operatorname{deg} \operatorname{lcm}(I: x)-\operatorname{ht}(I: x)+1 \leq \operatorname{deg} \operatorname{lcm}(I)-\text { ht } I .
$$

Therefore, the conclusion follows from Proposition 4.9.

\section{Depth and regularity functions}

In this section, we investigate the depth and the regularity of powers of an ideal. Our approach allows us to study the non-increasing and non-decreasing properties of the depth and the regularity functions for particular classes of ideals.

Let $I$ be a homogeneous ideal in a polynomial ring $R$. It is usually difficult to compute depth $R / I^{t}$ and reg $R / I^{t}$ for all $t \geq 1$. As a consequence, the behavior of depth $R / I^{t}$ and reg $R / I^{t}$, as functions in $t$, often remains mysterious. We only know that for $t$ sufficiently large, depth $R / I^{t}$ and reg $R / I^{t}$ are constant or a linear function, respectively $[4,8,24]$.

Random examples show that depth $R / I^{t}$ (respectively, reg $R / I^{t}$ ) tends to be a non-increasing (respectively, non-decreasing) function, though that is not the case in general $[1,2,14,15]$. Bandari, Herzog and Hibi [1] asked whether depth $R / I^{t}$ is a nonincreasing function for squarefree monomial ideals. A counter-example was recently found by combinatorial methods in [23] (see also [16]). However, it is still an open question whether depth $R / I^{t}$ is a non-increasing function for the edge ideal of an arbitrary graph.

Recall that a hypergraph is a collection of sets, called edges, which are subsets of a vertex set. Given a hypergraph $\mathcal{H}$ on the vertices $x_{1}, \ldots, x_{n}$, the edge ideal of $\mathcal{H}$ is defined to be

$$
\left.I(\mathcal{H}):=\left\langle\prod_{x \in F} x\right| F \text { is an edge of } \mathcal{H}\right\rangle .
$$

We will use Lemma 4.1(i) to present a large class of edge ideals of hypergraphs for which depth $R / I^{t}$ (respectively, reg $R / I^{t}$ ) is a non-increasing (respectively, nondecreasing) function. 
An edge $F$ of a hypergraph $\mathcal{H}$ is called a good leaf if the intersections of $F$ with other edges of $\mathcal{H}$ form a chain with respect to containment. This notion is originally introduced for simplicial complexes [20]. For the study of edge ideals, one may always assume that there are no containments among the edges of the hypergraph, which can be considered as the facets of a simplicial complex. The leaves of a graph are always good. Good leaves do exist in the hypergraph of the facets of a simplicial forest [20, Corollary 3.4], which may be defined as a hyperforest [20, Theorem 3.2].

Theorem 5.1 Let I be the edge ideal of a hypergraph which contains a good leaf. Then

(i) the functions depth $R / I^{t}$ and depth $R / \overline{I^{t}}$ are non-increasing for all $t \geq 1$,

(ii) the functions reg $R / I^{t}$ and $\operatorname{reg} R / \overline{I^{t}}$ are non-decreasing for all $t \geq 1$.

Proof Let $F$ be a good leaf and $f$ the monomial associated with $F$. Let $g$ be an arbitrary monomial of $I^{t+1}: f, t>0$. Then $f g=h f_{1} \cdots f_{t+1}$, where $h$ is a monomial and $f_{1}, \ldots, f_{t+1}$ are monomials associated with some edges $F_{1}, \ldots, F_{t+1}$ of the hypergraph. By the definition of a good leaf, we may assume that $F_{i} \cap F \subseteq F_{1} \cap F$ for all $i=1, \ldots, t+1$. Then the variables of $F \backslash F_{1}$ do not appear in $f_{1}, \ldots, f_{t+1}$. So they appear in $h$. From this it follows that $h f_{1}$ is divisible by $f$. Therefore, $g$ is divisible by $f_{2} \cdots f_{t+1}$. That means $g \in I^{t}$. Since $I^{t} \subseteq I^{t+1}: f$, we have shown that $I^{t+1}: f=I^{t}$. Hence, depth $R / I^{t} \geq \operatorname{depth} R / I^{t+1}$ and $\operatorname{reg} R / I^{t} \leq \operatorname{reg} R / I^{t+1}$ by Lemma 4.1.

Let $g \in \overline{I^{t+1}}: f$. Then $(g f)^{m} \in I^{m(t+1)}$ for some $m>0$. Similarly as above, we can show that $g^{m} \in I^{m t}$. Hence $g \in \overline{I^{t}}$. From this it follows that $\overline{I^{t+1}}: f=\overline{I^{t}}$. Since $\overline{I^{t}}$ is also a monomial ideal, depth $R / \overline{I^{t}} \geq \operatorname{depth} R / \overline{I^{t+1}}$ and $\operatorname{reg} R / \overline{I^{t}} \leq \operatorname{reg} R / \overline{I^{t+1}}$ by Lemma 4.1 .

In the following, we give formulas for depth $R /(I, f)^{s}$ and $\operatorname{reg} R /(I, f)^{s}$, when $f$ is a non-zerodivisor of $R / I^{t}$ for all $t \leq s$. The proof for these formulas follows the same line of arguments as that of Theorem 3.1.

Theorem 5.2 Let $R$ be a positively graded algebra over a field, and I be a graded ideal in $R$. Let $f$ be a form in $R$ which is a non-zerodivisor of $R / I^{t}$ for all $t \leq s$. Then

(i) depth $R /(I, f)^{s}=\min _{t \leq s} \operatorname{depth} R / I^{t}-1$,

(ii) $\operatorname{reg} R /(I, f)^{s}-s \operatorname{deg} f=\max _{t \leq s}\left\{\operatorname{reg} R / I^{t}-t \operatorname{deg} f\right\}$.

Proof Let $S=R[x]$ with $\operatorname{deg} x=\operatorname{deg} f=d$. If we consider $(I, x)$ as a graded module over $R$, we have a decomposition

$$
(I, x)^{s}=I^{s} \oplus I^{s-1} x \oplus \cdots \oplus I x^{s-1} \oplus R x^{s} \oplus R x^{s+1} \cdots
$$

From this, it follows that

$$
S /(I, x)^{S}=\left(R / I^{s}\right) \oplus\left(R / I^{s-1}\right)(-d) \oplus \cdots \oplus(R / I)(-(s-1) d) .
$$


Thus, we have

$$
\begin{aligned}
\operatorname{depth} S /(I, x)^{s} & =\min _{t \leq s} \operatorname{depth} R / I^{t}, \\
\operatorname{reg} S /(I, x)^{S}-s d & =\max _{t \leq s}\left\{\operatorname{reg} R / I^{t}-t d\right\} .
\end{aligned}
$$

Since $R /(I, f)^{S} \simeq R[x] /\left((I, x)^{s}, x-f\right)$, it remains to show that $x-f$ is a nonzerodivisor of $R[x] /(I, x)^{s}$. Let $g \in(I, x)^{s-1}$ such that $(x-f) g \in(I, x)^{s}$. Without restriction, we may assume that $g=c_{0}+c_{1} x+\cdots c_{s-1} x^{s-1}, c_{i} \in R$. Then $c_{0} f \in I^{s}$. Hence, $c_{0} \in I^{s}: f=I^{s}$. From this it follows that $\left(c_{1} x++\cdots c_{s-1} x^{s-1}\right)(x-f) \in$ $(I, x)^{s}$. This implies $c_{1} f \in I^{s-1}$. Hence, $c_{1} \in I^{s-1}: f=I^{s-1}$. Proceed in this fashion, we eventually get $c_{i} \in I^{s-i}$ for $i=0, \ldots, s-1$. Therefore, $g \in(I, x)^{s}$, as required.

Theorem 5.2 does not hold if $f \neq 0$ is not a nonzero divisor of $R / I^{t}$ for all $t \leq s$, as illustrated in the following example.

Example 5.3 Let $R=k[x, y, z, u, v]$ and consider

$$
I=\left(x^{5}, x^{4} y, x y^{4}, y^{5}, x^{2} y^{2}\left(x z^{6}-y u^{6}\right), x^{3} y^{3}\right) \text { and } f=z-u .
$$

Then $f$ is not a zero divisor of $R / I$, but $f$ is a zero divisor $R / I^{2}$.

We now have depth $R /(I, f)^{2}=1$, depth $R / I=2$, and depth $R / I^{2}=1$. Thus,

$$
\text { depth } R /(I, f)^{2} \neq \min _{1 \leq t \leq 2} \operatorname{depth} R / I^{t}-1 \text {. }
$$

We also have $\operatorname{reg} R /(I, f)^{2}=15, \operatorname{reg} R / I=10$, and $\operatorname{reg} R / I^{2}=20$. Therefore,

$$
\operatorname{reg} R /(I, f)^{2} \neq \max _{1 \leq t \leq 2}\left\{\operatorname{reg} R / I^{t}+(s-t) \operatorname{deg} f\right\} .
$$

From Theorem 5.2(i), we immediately obtain the following consequence.

Corollary 5.4 Let $R$ be a positively graded algebra over a field and I a graded ideal of $R$. Let $f$ be a form in $R$ which is not a zero divisor of $R / I^{t}$ for all $t \geq 0$. Then

(i) depth $R /(I, f)^{t}$ is a non-increasing function,

(ii) $\operatorname{reg} R /(I, f)^{t}-t \operatorname{deg} f$ is a non-decreasing function.

As an example, we may let $f$ be a new variable $x$, then depth $R[x] /(I, x)^{t}$ is a nonincreasing function and $\operatorname{reg} R[x] /(I, x)^{t}-t \operatorname{deg} f$ is a non-decreasing function. This displays an interesting phenomenon because the functions depth $R / I^{t}$ and $\operatorname{reg} R / I^{t}$ need not be so (see [1, Theorem 0.1], [14, Theorem 6.7] and [15, Example 2.8]).

Remark 5.5 Theorem 5.2(i) and Corollary 5.4(i) also hold for ideals in a local ring as it can be seen from the proof of Theorem 5.2(i). 
In the following, we denote by Ass $I$ the set of associated primes of $I$ in a Noetherian ring $R$. Brodmann [4] showed that Ass $I^{t}=$ Ass $I^{t+1}$ for $t \gg 0$. In general, we may have Ass $I^{t} \nsubseteq$ Ass $I^{t+1}$ (see, e.g., $[1,14,16]$ ). It has been of great interest to know when Ass $I^{t} \subseteq$ Ass $I^{t+1}$ for all $t \geq 1$ (see, e.g., [12,21,28]).

The following consequence of Theorem 5.2(i) shows that if there is a nonzero divisor $f$ modulo $I^{t}$ for all $t \geq 1$, then $\operatorname{Ass}(I, f)^{t} \subseteq \operatorname{Ass}(I, f)^{t+1}$ for all $t \geq 1$.

Corollary 5.6 Let $R$ be a Noetherian ring and I an ideal of $R$. Let $f \in R$ be an element which is not a zero divisor of $R / I^{t}$ for all $t \leq s$. Then $\operatorname{Ass}(I, f)^{t} \subseteq \operatorname{Ass}(I, f)^{t+1}$ for all $t<s$.

Proof Let $P \in \operatorname{Ass}(I, f)^{t}, t<s$. Then $\operatorname{depth}\left(R /(I, f)^{t}\right)_{P}=0$. By the local version of Theorem 5.2(i), we have $\operatorname{depth}\left(R / I^{j}\right)_{P}=1$ for some $j \leq t$, and hence

$$
\operatorname{depth}\left(R /(I, f)^{t+1}\right)_{P}=\min _{j \leq t+1} \operatorname{depth}\left(R / I^{j}\right)_{P}-1=0 .
$$

This implies that $P \in \operatorname{Ass}(I, f)^{t+1}$, as required.

\section{Depth and regularity of edge ideals}

The aim of this section is to show that our approach can be used to study edge ideals of hypergraphs. Throughout this section, we assume that all hypergraphs are simple and have no isolated vertices.

We will first investigate very well-covered graphs. Recall that a graph is wellcovered if all minimal vertex covers have the same size. A graph is very well-covered if all minimal vertex covers contain exactly half of the vertices. In these definitions, we can replace minimal vertex covers by maximal independent sets because they are complements of one another.

It is well known that every associated prime of the edge ideal of a graph is generated by the elements of a minimal vertex cover of the graph, and that this gives a correspondence between these two notions. Therefore, the edge ideal of a graph is (height) unmixed if and only if the graph is well-covered. An unmixed edge ideal of height $n$ in a polynomial ring of $2 n$ variables must come from a very well-covered graph. The interest in edge ideals of very well-covered graphs originates from bipartite graphs because an unmixed bipartite graph must be very well-covered.

A graph $\mathcal{H}$ is called Cohen-Macaulay if $R / I(\mathcal{H})$ is a Cohen-Macaulay ring, i.e., depth $R / I(\mathcal{H})=\operatorname{dim} R / I(\mathcal{H})$. It is well known that the edge ideal of a CohenMacaulay graph is always unmixed. Herzog and Hibi [18, Theorem 3.4] first gave a characterization of Cohen-Macaulay bipartite graphs. This result was extended to very well-covered graphs by Crupi, Rinaldo and Terai [7] (see also Constantinescu and Varbaro [6] and Mahmoudi et al [27]). We will give a short proof of their results. Our proof is based on the following effective characterizations of very well-covered graphs, due to Favaron [11].

Lemma 6.1 [11, Theorem 1.2] A graph $\mathcal{H}$ is very well-covered if and only if it has a perfect matching $M$ with the following two properties: 
(i) no edge of $M$ belongs to a triangle in $\mathcal{H}$,

(ii) if an edge of $M$ is the central edge of a path of length 3 in $\mathcal{H}$, then the two vertices at the ends of the path must be adjacent.

Moreover, if $\mathcal{H}$ is very well-covered, then every perfect matching in $\mathcal{H}$ satisfies these properties.

Recall that two vertices $x, y$ of a graph are twin if they have the same (open) neighborhood. A twin-free graph is sometimes referred to as irreducible by authors in graph theory.

Lemma 6.2 [11, Theorem 2.5] A very well-covered graph $\mathcal{H}$ is twin-free if and only if it has a perfect matching $M$ such that there is no cycle $C_{4}$ in $\mathcal{H}$ with two edges of $M$.

Our result on the Cohen-Macaulayness of very well-covered graphs is formulated in a different way than those of Constantinescu and Varbaro [6, Theorem 2.3], Crupi, Rinaldo and Terai [7, Theorem 3.4] and Mahmoudi et al [27, Theorem 3.2].

Theorem 6.3 Let $\mathcal{H}$ be a simple graph on $2 n$ vertices which has a minimal vertex cover of $n$ elements. Then $\mathcal{H}$ is a Cohen-Macaulay graph if and only if $\mathcal{H}$ is a twin-free very well-covered graph $\mathcal{H}$.

Proof Without restriction, we may assume that $\mathcal{H}$ is a very well-covered graph.

If $\mathcal{H}$ is not twin-free, then $\mathcal{H}$ has a cycle $C_{4}$ which contains two edges of a perfect matching of $\mathcal{H}$. It follows from Lemma 6.1(i) that the opposite vertices of this $C_{4}$ are not adjacent. Therefore, each maximal independent set in $\mathcal{H}$ contains exactly one pair of opposite vertices of this $C_{4}$. This implies that the independence complex of $\mathcal{H}$ is not strongly connected and, thus, is not Cohen-Macaulay; see [3, Proposition 11.7]. Hence, $\mathcal{H}$ is not Cohen-Macaulay.

Conversely, suppose that $\mathcal{H}$ is twin-free. Let $M=\left\{\left\{x_{1}, y_{1}\right\}, \ldots,\left\{x_{n}, y_{n}\right\}\right\}$ be a perfect matching in $\mathcal{H}$. Using Lemmas 6.1 and 6.2, it is easy to check that for any proper subset $K \subseteq[1, n]$, the induced subgraph of $\mathcal{H}$ on the vertices $\left\{x_{i}, y_{i}\right\}_{i \in K}$ is also a twin-free very well-covered graph. By induction, we may assume that these induced subgraphs are Cohen-Macaulay.

Consider a vertex $x$ in $\mathcal{H}$ and let $y$ be the vertex matched with $x$ in $M$. Let $N(x)$ denote the set of vertices in $\mathcal{H}$ adjacent to $x$. Observe that if there exists $z \in N(x)$ with $z \neq y$ then by Lemma 6.1(ii) we have $N(y) \subseteq N(z)$. Furthermore, this inclusion is strict since $y$ and $z$ are not twin. Thus, by taking $x$ such that $|N(y)|$ is largest possible, we may choose $x$ so that $N(x)=\{y\}$. Without loss of generality, suppose $N\left(x_{n}\right)=\left\{y_{n}\right\}$.

Let $R=k\left[x_{1}, \ldots, x_{n}, y_{1}, \ldots, y_{n}\right]$ and $I=I(\mathcal{H})$. Let $\mathcal{H}^{\prime}$ be the induced subgraph of $\mathcal{H}$ on $\left\{x_{1}, \ldots, x_{n-1}, y_{1}, \ldots, y_{n-1}\right\}$ and $R^{\prime}=k\left[x_{1}, \ldots, x_{n-1}, y_{1}, \ldots, y_{n-1}\right]$. Then $\left(I, y_{n}\right)=\left(I\left(\mathcal{H}^{\prime}\right), y_{n}\right)$. Since $R^{\prime} / I\left(\mathcal{H}^{\prime}\right)$ is a Cohen-Macaulay ring with $\operatorname{dim} R^{\prime} / I\left(\mathcal{H}^{\prime}\right)=n-1$, we have

$$
\operatorname{depth} R /\left(I, y_{n}\right)=\operatorname{depth} S / I\left(\mathcal{H}^{\prime}\right)+1=n \text {. }
$$

Without loss of generality, we may assume that $\left\{y_{1}, \ldots, y_{n}\right\}$ is a maximal independent set of $\mathcal{H}$. Suppose $N\left(y_{n}\right)=\left\{x_{i_{1}}, \ldots, x_{i_{s}}\right\}$. Let $\mathcal{G}$ denote the induced 
subgraph of $\mathcal{H}$ on $Z:=\left\{x_{i}, y_{i} \mid i \neq i_{1}, \ldots, i_{s}\right\}$ and $S=k[Z]$. By Lemma 6.1(ii), the vertices $y_{i_{1}}, \ldots, y_{i_{s}}$ are not adjacent to any vertex $z \in Z$. This implies that $\left(I: y_{n}\right)=I(\mathcal{G})+\left(x_{i_{1}}, \ldots, x_{i_{s}}\right)$. Since $S / I(\mathcal{G})$ is a Cohen-Macaulay ring with $\operatorname{dim} S / I(\mathcal{G})=n-s$, we have

$$
\operatorname{depth} R /\left(I: y_{n}\right)=\operatorname{depth} S / I(\mathcal{G})+s=n \text {. }
$$

By Theorem 4.3, we have depth $R / I \in\left\{\operatorname{depth} R /\left(I: y_{n}\right)\right.$, depth $\left.R /\left(I, y_{n}\right)\right\}$. Therefore, $\operatorname{depth} R / I=n=\operatorname{dim} R / I$.

We shall now discuss an application of Proposition 4.9 on the regularity of edge ideals. For a hypergraph $\mathcal{H}$, we set $\operatorname{reg} \mathcal{H}=\operatorname{reg} I(\mathcal{H})$ and call it the regularity of $\mathcal{H}$.

If reg $\mathcal{H} \leq 2$, the degree of the minimal monomial generators of $I(\mathcal{H})$ is bounded by 2 . Hence, $I(\mathcal{H})$ is actually the edge ideal of a graph. In particular, $\mathcal{H}$ is a graph if there are no containments among the edges. If $\mathcal{H}$ is a graph, we know that reg $\mathcal{H} \leq 2$ if and only if the complement of $\mathcal{H}$ is chordal [13]. Recall that a graph is called chordal if it has no induced cycle $C_{m}$ with $m \geq 4$. In the following, we give a necessary condition for reg $\mathcal{H} \leq 3$.

For a vertex $x$ of a hypergraph $\mathcal{H}$, we denote by $\mathcal{H}: x$ the hypergraph of all minimal sets of the form $F \cap(V \backslash\{x\})$, where $F$ is an edge of $\mathcal{H}$.

Theorem 6.4 Let $\mathcal{H}$ be a hypergraph such that $\mathcal{H}: x$ is a graph whose complement is chordal for all vertices $x$ of $\mathcal{H}$. Then $\operatorname{reg} \mathcal{H} \leq 3$.

Proof Let $I=I(\mathcal{H})$. Then $I: x=I(\mathcal{H}: x)$ for all vertices $x$ of $\mathcal{H}$. By the above result of Fröberg, the assumption on $\mathcal{H}: x$ implies that $\operatorname{reg}(I: x) \leq 2$. Therefore, $\operatorname{reg} I \leq 3$ by Proposition 4.9 .

The above necessary condition for reg $\mathcal{H} \leq 3$ is not so far from being a sufficient condition because due to Corollary $4.8, \operatorname{reg} \mathcal{H} \leq 3$ if and only if $\operatorname{reg}(\mathcal{H}-x) \leq 3$ or $\operatorname{reg}(\mathcal{H}: x) \leq 2$ for some vertex $x$. Here, $\mathcal{H}-x$ denotes the hypergraph of the edges of $\mathcal{H}$ not containing $x$. If $\operatorname{reg}(\mathcal{H}: x) \leq 2$, then $\mathcal{H}: x$ is a graph (there are no containments among the edges of $\mathcal{H}: x$ ) whose complement is chordal by [13].

As a consequence, we obtain the following result of Nevo [30, Theorem 5.1], which was also reproved by Dao, Huneke and Schweig [9, Theorem 3.4]. Recall that a claw of a graph is an induced complete bipartite graph $K_{1,3}$ and a gap is an induced graph whose complement is a cycle $C_{4}$.

Corollary 6.5 Let $\mathcal{H}$ be a claw-free and gap-free graph. Then reg $\mathcal{H} \leq 3$.

Proof By Theorem 6.4, we only need to show that the complement of $\mathcal{H}: x$ is a chordal graph for all $x \in V$. Assume that there is a vertex $x$ such that the complement of $\mathcal{H}: x$ is not chordal. Then the complement of $\mathcal{H}: x$ contains an induced cycle $C_{m}, m \geq 4$. Let $G$ denote the induced subgraph of $\mathcal{H}$ whose complement is this $C_{m}$. Let $y$ be an adjacent vertex of $x$. Then $y$ is not a vertex of $G$. If $y$ were not adjacent to an edge of $G$, the induced graph on $x, y$ and the vertices of this edge were a gap. Therefore, $y$ must be adjacent to every edge of $G$. Let $z$ be a vertex of $G$ adjacent to $y$. Let $u, v$ be the two vertices of $G$ non-adjacent to $z$. Then $\{u, v\}$ is an edge of $G$. 
Hence, $y$ is adjacent to one of them, say $u$. Since $y$ is adjacent to $x, z, u$ and since $x, z, u$ are not adjacent to each other, the induced subgraph on $x, y, z, u$ is a claw, a contradiction.

Acknowledgements Giulio Caviglia is partially supported by the Simons Foundation (Grant \#209661). Huy Tài Hà is partially supported by the Simons Foundation (Grant \#279786). Ngo Viet Trung is partially supported by Vietnam National Foundation for Science and Technology Development (Grant \#101.042017.19) and the project VAST.HTQT.NHAT.1/16-18. The main part of this work was done during research stays of the authors at the American Institute of Mathematics in the SQuaRE program "Ordinary powers and symbolic powers" during the period 2012-2014. The authors are grateful to S. A. Seyed Fakhari for pointing out a mistake of Theorem 5.1 in the first version of this paper and to an anonymous referee for mentioning that Lemma 4.1(i) and (ii) were already proved in [31] and [32], respectively.

\section{References}

1. Bandari, S., Herzog, J., Hibi, T.: Monomial ideals whose depth function has any given number of strict local maxima. Ark. Mat. 52, 11-19 (2014)

2. Berlekamp, D.: Regularity defect stabilization of powers of an ideal. Math. Res. Lett. 19, 109-119 (2012)

3. Björner, A.: Topological methods. In: Graham, R.L., Grötschel, M., Lovász, L. (eds.) Handbook of Combinatorics, vol. 2, pp. 1819-1872. Elsevier, Amsterdam (1995)

4. Brodmann, M.: The asymptotic nature of the analytic spread. Math. Proc. Camb. Philos. Soc. 86, 35-39 (1979)

5. Bruns, W., Herzog, J.: Cohen-Macaulay Rings. Cambridge University Press, Cambridge (1993)

6. Constantinescu, A., Varbaro, M.: On the $h$-vectors of Cohen-Macaulay flag complexes. Math. Scand. 112, 86-111 (2013)

7. Crupi, M., Rinaldo, G., Terai, N.: Cohen-Macaulay edge ideals whose height is half of the number of vertices. Nagoya Math. J. 201, 116-130 (2011)

8. Cutkosky, S.D., Herzog, J., Trung, N.V.: Asymptotic behaviour of the Castelnuovo-Mumford regularity. Compos. Math. 118(3), 243-261 (1999)

9. Dao, H.L., Huneke, C., Schweig, J.: Bounds on the regularity and projective dimension of ideals associated to graphs. J. Algebr. Comb. 38(1), 37-55 (2013)

10. Eisenbud, D.: Commutative Algebra: With a View Toward Algebraic Geometry. Springer, New York (1995)

11. Favaron, O.: Very well-covered graphs. Discrete Math. 42(2-3), 177-187 (1982)

12. Francisco, C.A., Hà, H.T., Van Tuyl, A.: Colorings of hypergraphs, perfect graphs, and associated primes of powers of monomial ideals. J. Algebra 331, 224-242 (2011)

13. Fröberg, R.: On Stanley-Reisner rings. In: Balcerzyk, S., Józefiak, T., Krempa, J., Simson, D., Vogel, W. (eds.) Topics in Algebra. Banach Center Publications, vol. 26(2), pp. 57-70 (1990)

14. Hà, H.T., Nguyen, H.D., Trung, N.V., Trung, T.N.: Symbolic powers of sums of ideals. Preprint arXiv: 1702.01766

15. Hà, H.T., Trung, N.V., Trung, T.N.: Depth and regularity of powers of sums of ideals. Math. Z. 282(3-4), 819-838 (2016)

16. Hà, H.T., Sun, M.: Squarefree monomial Ideals that fail the persistence property and non-increasing depth. Acta Math. Vietnam. 40, 125-137 (2015)

17. Herzog, J., Hibi, T.: The depth of powers of an ideal. J. Algebra 291, 534-550 (2005)

18. Herzog, J., Hibi, T.: Distributive lattices, bipartite graphs and Alexander duality. J. Algebr. Comb. 22(3), 289-302 (2005)

19. Herzog, J., Hibi, T.: Monomial Ideals. Springer, Berlin (2011)

20. Herzog, J., Hibi, T., Trung, N.V., Zheng, X.: Standard vertex cover algebras, cycles and leaves. Trans. Am. Math. Soc. 360(12), 6231-6249 (2008)

21. Herzog, J., Qureshi, A.A.: Persistence and stability properties of powers of ideals. J. Pure Appl. Algebra 219(3), 530-542 (2015)

22. Hoa, L.T., Trung, N.V.: On the Castelnuovo-Mumford regularity and the arithmetic degree of monomial ideals. Math. Z. 229(3), 519-537 (1998) 
23. Kaiser, T., Stehlík, M., Skrekovski, R.: Replication in critical graphs and the persistence of monomial ideals. J. Comb. Theory Ser. A 123, 239-251 (2014)

24. Kodiyalam, V.: Asymptotic behaviour of Castelnuovo-Mumford regularity. Proc. Am. Math. Soc. 128(2), 407-411 (1999)

25. Kummini, M.: Homological invariants of monomial and binomial ideals. Thesis, University of Kansas (2008)

26. Kummini, M.: Regularity, depth and arithmetic rank of bipartite edge ideals. J. Algebr. Comb. 30, 429-445 (2009)

27. Mahmoudi, M., Mousivand, A., Crupi, M., Rinaldo, G., Terai, N., Yassemi, S.: Vertex decomposability and regularity of very well-covered graphs. J. Pure Appl. Algebra 215, 2473-2480 (2011)

28. Martinez-Bernal, J., Morey, S., Villarreal, R.H.: Associated primes of powers of edge ideals. Collect. Math. 63(3), 361-374 (2012)

29. Minh, N.C., Trung, N.V.: Cohen-Macaulayness of monomial ideals and symbolic powers of StanleyReisner ideals. Adv. Math. 226, 1285-1306 (2011)

30. Nevo, E.: Regularity of edge ideals of $C_{4}$-free graphs via the topology of the lcm-lattice. J. Comb. Theory Ser. A 118, 491-501 (2011)

31. Rauf, A.: Depth and Stanley depth of multigraded modules. Commun. Algebra 38, 773-784 (2010)

32. Seyed Fakhari, S.A.: Symbolic powers of cover ideal of very well-covered and bipartite graphs. Proc. Am. Math. Soc. 146, 97-110 (2018)

33. Takayama, Y.: Combinatorial characterizations of generalized Cohen-Macaulay monomial ideals. Bull. Math. Soc. Sci. Math. Roum. (N.S.) 48, 327-344 (2005)

34. Trung, N.V.: Reduction exponent and degree bounds for the defining equations of a graded ring. Proc. Am. Math. Soc. 101, 229-236 (1987)

35. Trung, N.V.: Gröbner bases, local cohomology and reduction number. Proc. Am. Math. Soc. 129, 9-18 (2001) 